\title{
Estrés docente y factores psicosociales en docentes de Latinoamérica, Norteamérica y Europa
}

\section{Teacher Stress and Psychosocial Factors in Teachers from Latin America, North America and Europe}

Cleofé Genoveva Alvites-Huamaní

Universidad Alas Peruanas, Lima, Perú

ORCID: https://orcid.org/0000-0001-6328-6470

Recibido 26-07-19 Revisado 05-08-19 Aprobado 23-09-19 En línea 02-10-19

*Correspondencia

Email: cleoalvitesh@gmail.com
Citar como:

Alvites-Huamaní, C. (2019). Estrés docente y factores psicosociales en docentes de Latinoamérica, Norteamérica y Europa. Propósitos y Representaciones, 7(3), 141-178. doi: http://dx.doi.org/10.20511/pyr2019.v7n3.393 


\section{Resumen}

En un mundo acelerado como el de estos tiempos, en el que la información y el conocimiento cambia en milésimas de segundos y por ende la educación a todo nivel debe adaptarse a ella, esta como centro el docente, quien debe realizar todas sus actividades con urgencia por presión mediática que tiene de su entorno, autoridades, pares y hasta de sus estudiantes, lo cual en el transcurso del tiempo le puede generar estrés, por tanto esta investigación se centró en relacionar el estrés y los factores psicosociales en docentes de Latinoamérica, Norteamérica y Europa, en una muestra no probabilística $n=81$ docentes de diferentes países, del nivel de educación básica y universitaria, para la recolección de datos se utilizaron la Escala Docente (ED-6 $\alpha=0.932$ ) y Cuestionario de Factores Psicosociales en el Trabajo Académico (FPSIS $\alpha=0.916$ ), con un diseño no experimental, correlacional, transversal de enfoque cuantitativo. Los resultados obtenidos indican que existe correlación significativa entre el estrés docente y los factores psicosociales, $(p=.000)$, prevaleciendo en su mayoría el nivel de estrés por ansiedad, depresión, creencias desadaptativas que se correlacionan con los factores psicosociales.

Palabras Clave: Depresión; Estrés docente; Ansiedad; Factores psicosociales; Educación.

\section{Summary}

In a fast-paced world like that of these days, in which information and knowledge change in thousandths of seconds, and therefore education at all levels must adapt to this. Teachers, who are at the center, must carry out all their activities urgently due to media pressure in their environment, the pressure from authorities, coworkers and even their students. This can lead to stress over time, that is why this research focused on relating stress and psychosocial factors in teachers from Latin America, North America and Europe, involving in a non-probabilistic sample $n=81$ teachers from different countries, at basic education and university levels. The Teaching Scale (ED-6 $\alpha=$ 0.932) and the Questionnaire of Psychosocial Factors at Academic Work (FPSIS $\alpha=0.916$ ) were used for the data collection, with a non-experimental, correlational, cross-sectional to quantitative approach design. The results obtained indicate that there is a significant correlation between teacher stress and psychosocial factors, $(\mathrm{p}=.000)$, prevailing mostly the level of stress due to anxiety, depression, and non-adaptive beliefs that correlate with the psychosocial factors.

Keywords: Depression; Teacher Stress; Anxiety; Psychosocial Factors; Education.

\section{Introducción}

Las exigencias que en este siglo demanda la globalización aunado al incesante incremento de las tecnologías de la información y la comunicación, las cuales han repercutido en cambios sustanciales en el mercado laboral, la economía, y de manera muy particular en la educación básica y universitaria, (Parihuaman-Aniceto, 2017; García-Moran \& Gil-Lacruz, 2016), ya que esta se ha visto obligada a realizar drásticos cambios para cumplir con los estándares de una educación de alta calidad, debido a que en estos últimos tiempos los gobiernos y entes responsables exigen una mayor transparencia de como se está formando a los futuros profesionales que se integrarán al mercado laboral, y por ende, ha conllevado a que una ingente cantidad de instituciones educativas se vean obligadas a exigir a su plana docente, adicional a las horas académicas programadas, brinden horas como apoyo administrativo, interacción con los padres de familia y tutoría a los estudiantes, que aunque no le compete algunas de estas actividades, está obligado a realizarla -al cumplir este un rol fundamental en el proceso enseñanza - aprendizaje- y por haber sido asignado por las autoridades de sus instituciones educativas, para González, (2018); Chavarria et al., (2017) estos nuevos roles mencionados están relacionados con la sobrecarga de trabajo y se vinculan directamente con el cansancio emocional, síntomas de 
ansiedad y depresión, lo cual deriva en estrés en el docente, (Parihuaman-Aniceto, 2017; Castillo, Fernández y López, 2014). El estrés es una da las causas a nivel mundial que deriva en absentismo, falta de compromiso con la institución, insatisfacción en el centro de trabajo y genera en el que la padece depresión y ansiedad (García-Rivera, Maldonado-Radillo y Ramírez, 2014; Reyes \& Aguilar, 2015). Según Zuñiga-Jara y Pizarro-León, (2018); González, (2018); Castro, (2008), refieren que el docente al tener que lidiar con un excesivo número de estudiantes en sus aulas, indisciplina, falta de interés por aprender, bajo apoyo de padres, el abuso de poder de sus directores o jefe inmediato, presentación de informes y otros tipos de documentos, conlleva a generarles estrés, el cual puede incidir negativamente en su desempeño docente. De acuerdo con González (2018), el estrés que puede padecer un docente está también relacionado con factores psicosociales, dentro del cual la infraestructura, el equipamiento, los espacios físicos, la tecnología inciden en la afectación de su salud emocional, debido a que estos no están diseñados adecuadamente o no han sido capacitados para el uso de estos medios. Además, la docencia es una profesional que exige mucho a los que la ejercen, al tener que estar al contacto con personas con una variedad de costumbres, ideologías, cultura, lo cual implica una adaptación constante por parte de ellos a estas situaciones cambiantes en este colectivo y en la sociedad misma, lo que también les genera estrés (Perez, 2018). Desde esta perspectiva es relevante hacer estudios sobre la problemática expuesta, que permitan evaluar si estos tienen alguna incidencia en los docentes de todos los niveles educativos, por lo que se planteó como objetivo general determinar la relación entre estrés docente y factores psicosociales en docentes de Latinoamérica, Norteamérica y Europa, y, como objetivos específicos: (i) determinar la relación entre el estrés y factores psicosociales en la dimensión condiciones del lugar de trabajo en los docentes, (ii) determinar la relación entre el estrés y los factores psicosociales en la dimensión carga de trabajo en los docentes, (iii) determinar la relación significativamente con los factores psicosociales en la dimensión contenido y característica de la tarea en los docentes (iv) determinar la relación entre estrés y factores psicosociales en la dimensión exigencias laborales en los docentes. (v.) determinar la relación entre estrés y factores psicosociales en la dimensión papel del académico y desarrollo de la carrera en los docentes. (vi.) determinar la relación entre el estrés y factores psicosociales en la dimensión interacción social y aspectos organizacionales en los docentes. (vii.) determinar la relación entre estrés y factores psicosociales en la dimensión remuneración de rendimiento en los docentes de Latinoamérica, Norteamérica y Europa.

\section{Estrés docente}

El estrés en general es una afectación que el ser humano ha padecido desde siglos pasados, González, (2018); Osorio \& Cárdenas, (2017); Uribe, Patlán \& García, (2014) refieren que es una respuesta automática del organismo ante cualquier acontecimiento que se le imponga y que sienta que es amenazadora, haciendo que el sistema nervioso se estimule y reaccione produciendo cambios a nivel psicológico (mental) o fisiológico (físico), y que se da de manera particular entre la persona y la situación. Para Sánchez (2017) se distingue el estrés del estrés laboral, el cual acontece del desequilibrio de las exigencias y presiones que enfrenta el individuo por presiones en el trabajo y condiciones laborales no óptimas, las cuales exceden la resistencia y capacidad del trabajador para poder resolver y enfrentar estas, Parihuaman-Aniceto, (2017). Para Orozco, (2018); Chiang, Riquelme \& Rivas, (2018); Zuñiga-Jara y Pizarro-León, (2018); Cardozo, (2016); Barradas et al., (2015); Díaz, (2011) el estrés laboral es una respuesta fisiológica, emocional y conductual, que lleva a una pérdida de motivación, e influye hacia sentimientos de inadecuación y fracaso en el trabajador, lo cual le genera un desajuste en su desempeño y repercute en su salud física y psicológica, mermando en su capacidad de concentración, cambios fluctuantes en su estado de ánimo, llegando hasta padecer depresión (Chiang, Heredia \& Santamaría, 2017; Donayre, 2016). El estrés no distingue ni raza, ni estatus económico y mucho menos la profesional, por lo que los docentes no son ajenos a padecer de este, como lo menciona De la Cruz, (2017); García-Moran \& Gil-Lacruz, (2016) al referir que el estrés docente es una angustia que no se disipa y que produce en él reacciones adversas en su organismo, para lo cual utiliza mecanismos fisiológicos (taquicardia, insomnio, trastornos digestivos, hipertensión), psicológicos (irritabilidad, ansiedad, ira, depresión) y mecanismos conductuales (aburrimiento, 
divagación, abuso de tabaco, alcohol, drogas) para reaccionar, ante estos cambios que se manifiesta por meses generándole agotamiento y desgaste mermando en su salud, manifestando según Cardozo, (2016); Castro, (2008) en emociones negativas y desagradables como enfado, ansiedad, frustración, depresión, angustia y nerviosismo. Adicional a ello, González, (2018); De la Cruz (2017); Sánchez, (2017), Patiño y Gutiérrez, (2013) inciden en que el estrés docente implica el que este no pueda tener un desempeño laboral óptimo y de calidad, debido a la afectación de falta de energía y entusiasmo que tenía antes para lograr sus metas y cumplir con sus diversas actividades, al haberse visto obligado a adoptar hábitos distintos a los que estaba acostumbrado y enfrentarse a estos mecanismos fisiológicos, psicológicos y conductuales.

Para Espadas, (2016); Castro, (2008) los cambios que se están realizando constantemente a nivel de estándares en la educación y el reconocer a las institucione educativas como acreditadas, hacen que estas incidan en los docentes con una mayor presión laboral, carga de trabajo y funciones adicionales dentro de las horas académicas asignadas, sin ningún beneficio adicional, continuos cambios en los contenidos curriculares, incremento de la conflictividad en el aula, mayor cantidad de estudiantes con necesidades especiales, influencia de las medios tecnológicos, lo cual ha demandado en ellos el tener que estar muchas más horas de las planificadas en su centro de trabajo, dejando de lado otras obligaciones a realizar, y como consecuencia se deriva en estrés con manifestaciones psicológicas como la ansiedad basadas en creencias desadaptativas, depresión con alteraciones del humor, apatía, tristeza, que pueden llegar a incidir en conductas no deseables por parte de los docentes, Parihuaman-Aniceto, (2017) y con un mal afrontamiento a estas situaciones estresantes repercutiendo en el maltrato a sus estudiantes y a las personas que están en su entorno, como también en un deterioro cognitivo al afectar su concentración, y atención lo que se refleja en una disminución en su rendimiento académico (Sánchez, 2017; Cardozo, 2016).

\section{Factores psicosociales y Estrés docente}

En este siglo XXI donde los cambios son constantes sobre todo en el ámbito laboral, donde las exigencias de nuevos perfiles se ha ido acentuando, así como no tener un horario establecido de horas de trabajo, a pesar que esto si lo indica en el contrato de los profesionales, esto no se refleja en la realidad, junto con el cual las condiciones del lugar de trabajo no son las más idóneas para el desempeño de sus actividades, con una excesiva carga laboral, imposibilitando el desarrollo profesional del trabajador en el ámbito académico y educación continua aunado a ello un salario no acorde con las expectativas de los profesionales o con las funciones que realiza, conlleva a que estos factores psicosociales influyen en detrimento de la salud del trabajador y sobre todo en la productividad de la organización, disminución del rendimiento y de la calidad, absentismo y abandono, (Fernández-Puig et al., 2015), no siendo consciente de las perdidas que le genera a su institución que su personal este padeciendo agotamiento emocional o estrés, que conllevan a depresión y ansiedad, como lo refiere Montiel-Jarquín et al., (2015) al tener jornadas largas de trabajo, un inadecuado tiempo de descanso, instalaciones precarias o en mal estado de conservación, carencia de materiales y equipo, baja remuneración. Chavarría et al., (2016); Castillo, Fernández y López, (2014), mencionan que la docencia actualmente es reconocida como una profesión altamente estresante al tener estos que enfrentarse a un gran conflicto en sus actividades, debido al incremento de tareas en las que no fueron capacitados, el pobre reconocimiento que tienen, el tener una sobrecarga laboral, realizar funciones administrativas y cambios de roles a los que son sometidos constantemente. Sánchez, (2017) refiere que hay factores sociolaborales que inciden en el docente, como es la condición laboral que en algunos casos son contratados por un periodo corto y otros que gozan de estabilidad laboral, son estos los que tienen mayores niveles de estrés; además, presentan sobrecarga en el trabajo y tienen escaso apoyo social lo que repercute en una insatisfacción y estrés. Según Perez, (2018); Chiang, Heredia y Santamaria, (2017); Díaz, (2011) los factores psicosociales son el conjunto de condiciones propias del individuo, de su medio laboral y de su entorno extralaboral, el nivel de presión, el volumen de trabajo, la estabilidad laboral, reconocimiento, elevada carga laboral, si estas repercuten negativamente de manera intensa y de exposición prolongada produce efectos 
negativos y desajustes en su salud a nivel intelectual, fisiológico, psicoemocional y social que desencadena en estrés. Para Osorio y Cárdenas, (2017) los factores psicosociales son las condiciones intralaborales como las extralaborales del individuo que influyen en la aparición de estrés cuando estos se vuelven un riesgo, como el aumento de responsabilidades, actividades repetitivas que se realiza de manera frecuente y por periodos largos, la inestabilidad laboral y trabajo excesivo, Lemos et al., (2018); González, (2018). Villanueva et al., (2005) enfatiza que existen factores sociales que inciden en el estrés docente, como la perdida de autoridad frente a los padres de familia, desmotivación y actitudes de indisciplina por parte de los estudiantes, perdida de estatus y prestigio.

Fernández-Puig et al., (2015) mencionan que los factores psicosociales son la principal causa de pérdida de salud en los docentes y que esto se manifiesta a través del estrés crónico y además son propensos a aumentar el riesgo de sufrir alteraciones emocionales, cognitivas y psicosomáticas. De igual manera, Unda, et al., (2016) refieren que la Organización Internacional del Trabajo menciona que los factores psicosociales en el ámbito laboral contribuyen a una serie de daños para la salud si estos son inadecuados o de riesgo para el trabajador pudiendo tener una afectación de carácter psicológico, comportamental, somáticos o emocionales causantes de estrés, depresión y dolor, resaltando en el caso de los profesionales maestros, médicos, enfermeras, entre otros. Según Graneros, (2018) la interacción que se da entre el trabajador y el ambiente físicosocial, enmarcados dentro de factores psicosociales, puede afectar su salud si esta sometido a tensiones o estrés, debido a que no todos los trabajadores afrontan del mismo modo situaciones estresantes, porque cada uno tiene características propias que determinan la magnitud y naturaleza de sus reacciones. El ambiente físico debe tener una adecuada iluminación para el desempeño de las actividades del trabajador para que no le genere fatiga, el ruido repercute negativamente, si este pasa los decibeles permitidos para un individuo, así como el espacio de trabajo debe cumplir las medidas normadas, ya que si este es reducido el trabajador se ve obligado a mantener solo una misma postura por periodos largos, estos aspectos negativos pueden desencadenar el estrés (Graneros, 2018).

\section{Método}

\section{Participantes}

La población estuvo compuesta por 329 docentes de educación básica y educación superior de Latinoamérica, (Argentina, Bolivia, Perú, Colombia, Uruguay, Venezuela, Panamá y México), de Norteamérica (Estados Unidos) y Europa (España). Al ser un estudio exploratorio la muestra fue de tipo no probabilística, intencional, según Hernández, Fernández y Baptista, (2014) la selección de los sujetos no depende de la probabilidad, sino a los propósitos del investigador, teniendo como criterio de inclusión la participación voluntaria con la aceptación del consentimiento informado, docentes en actividad con asignación de por lo menos un aula, por lo que el total de la muestra fue de 81 docentes, predominando la edad entre 41 y 50 años (36\%); en cuanto al nivel de estudios el grado de magister es en mayor porcentaje (48\%), la mayoría es casado (56\%), residen en Latinoamérica (88\%), Norteamérica (7\%) y en Europa (5\%), lo cual se describe en la tabla 1. En cuanto al nivel educativo donde labora (52\%) lo realiza en la universidad, en una institución pública (75\%), tienen a su cargo entre 1 y 3 aulas (59\%), mayormente enseñan en el turno mañana (70\%) y se dedican a la enseñanza más de 11 años (73\%), como se describe en la tabla 2. 
Tabla 1.

Descripción 1 de la muestra en porcentajes

\begin{tabular}{cccccccc}
\hline Edad & $\begin{array}{c}\% \text { de } \\
\text { docentes }\end{array}$ & $\begin{array}{c}\text { Nivel de } \\
\text { estudios }\end{array}$ & $\begin{array}{c}\% \text { de } \\
\text { docentes }\end{array}$ & Estado civil & $\begin{array}{c}\% \text { de } \\
\text { docentes }\end{array}$ & $\begin{array}{c}\text { País de } \\
\text { residencia }\end{array}$ & $\begin{array}{c}\% \text { de } \\
\text { docentes }\end{array}$ \\
\hline $\begin{array}{c}\text { 20 a } 30 \\
\text { años }\end{array}$ & 1 & Bachiller & 3 & Casado & 56 & $\begin{array}{c}\text { Latinoaméric } \\
\text { a }\end{array}$ & 88 \\
$\begin{array}{c}\text { 31 a } 40 \\
\text { años }\end{array}$ & 21 & $\begin{array}{c}\text { Licenciad } \\
\text { o }\end{array}$ & 16 & Soltero & 22 & Norteamérica & 7 \\
$\begin{array}{c}41 \text { a } 50 \\
\text { años }\end{array}$ & 36 & $\begin{array}{c}\text { Magister } \\
\text { añ a } 60\end{array}$ & 48 & Divorciado & 9 & Europa & 5 \\
$\begin{array}{c}\text { años } \\
\text { 61 a }\end{array}$ & 11 & Doctor & 32 & Conviviente & 12 & & \\
más & & Otro & 1 & Viudo/a & 1 & & \\
años & & & & & & & \\
\hline
\end{tabular}

Tabla 2.

Descripción 2 de la muestra en porcentajes

\begin{tabular}{|c|c|c|c|c|c|c|c|c|c|}
\hline $\begin{array}{c}\text { Nivel } \\
\text { educativo } \\
\text { de trabajo }\end{array}$ & $\begin{array}{c}\% \\
\text { de } \\
\text { docentes }\end{array}$ & $\begin{array}{c}\text { Tipo de } \\
\text { institució } \\
n\end{array}$ & $\begin{array}{c}\% \\
\text { de } \\
\text { docentes }\end{array}$ & $\begin{array}{l}\mathrm{N}^{\circ} \text { de } \\
\text { aulas }\end{array}$ & $\begin{array}{c}\% \\
\text { de } \\
\text { docentes }\end{array}$ & $\begin{array}{l}\text { Turno de } \\
\text { enseñanza }\end{array}$ & $\begin{array}{c}\% \\
\text { de } \\
\text { docentes } \\
\end{array}$ & $\begin{array}{c}\begin{array}{c}\text { Años } \\
\text { de } \\
\text { enseñanza }\end{array} \\
\end{array}$ & $\begin{array}{c}\% \\
\text { de } \\
\text { docentes }\end{array}$ \\
\hline Inicial & 5 & Pública & 75 & $\begin{array}{l}1 \text { a } 3 \\
\text { aulas }\end{array}$ & 59 & Mañana & 70 & $\begin{array}{l}1 \text { a } 5 \\
\text { años }\end{array}$ & 9 \\
\hline Primaria & 26 & Privada & 22 & $\begin{array}{l}4 \text { a } 6 \\
\text { aulas }\end{array}$ & 21 & Tarde & 14 & $\begin{array}{c}5 \text { a } 10 \\
\text { años }\end{array}$ & 18 \\
\hline $\begin{array}{l}\text { Secunda } \\
\text { ria }\end{array}$ & 11 & $\begin{array}{c}\text { Pública } \\
y \\
\text { privada }\end{array}$ & 3 & $\begin{array}{l}\text { Más } \\
\text { de } 6 \\
\text { aulas }\end{array}$ & 20 & Noche & 16 & $\begin{array}{l}\text { Más de } \\
11 \text { años }\end{array}$ & 73 \\
\hline Instituto & 6 & & & & & & & & \\
\hline $\begin{array}{c}\text { Universi } \\
\text { dad }\end{array}$ & 52 & & & & & & & & \\
\hline
\end{tabular}

\section{Instrumento}

Para la recolección de los datos de la variable estrés docente se utilizó la Escala de Estrés Docente ED-6, elaborada por Gutiérrez, Morán, Sanz e Inmaculada (Orozco, 2018), la cual fue aplicada en población peruana por Parihuaman-Aniceto, (2017), el cual constaba de 55 ítems, pero para este estudio se adaptó debido a que la muestra incluía tres tipos de contextos diferentes. Al ser adaptada la ED-6 se realizó la validez de contenido por juicio de expertos, siendo cuatro expertos en el tema que residen en México, Panamá, Estados Unidos y Perú, quienes evaluaron el instrumento, sugiriendo pequeños cambios y eliminación de algunos ítems, que se consideró pertinente tenerlo en cuenta, lo que se puede observar en el anexo 1. La confiabilidad del ED-6 se verificó con el Alfa de Cronbach (tabla 5) obteniendo $\alpha=0.932$ (Muy alta confiabilidad). El ED-6, (tabla 3) posee 5 dimensiones y 39 ítems tipo escala de Likert, con una valoración de 1 (totalmente de acuerdo) a 5 (Totalmente en desacuerdo); autoaplicable en un tiempo aproximado de 25 minutos de administración colectiva. 
Tabla 3.

Descripción del instrumento Estrés docente ED-6

\begin{tabular}{llc}
\hline \multicolumn{1}{c}{ Dimensiones } & \multicolumn{1}{c}{ Ítem } & Instrumento \\
\hline Estrés por ansiedad & $1,2,3,4,5,6,7,8,9,10$ & \\
Estrés por depresión & $11,12,13,14,15,16,17$ & $\begin{array}{c}\text { Escala de Estrés } \\
\text { docente }\end{array}$ \\
Estrés por creencias desadaptativas & $18,19,20,21$ & (ED-6) \\
Estrés por presión laboral & $22,23,24,25,26,27,28,29,30$, & \\
Estrés por mal afrontamiento & $31,32,33,34$ & \\
& $35,36,37,38,39$ & \\
\hline
\end{tabular}

Para la variable factores psicosociales (tabla 4), se utilizó el Cuestionario de Factores Psicosociales en el Trabajo Académico (FPSIS) elaborado por Blanca Silva Gutiérrez en el 2005, el cual fue aplicado en población peruana por Graneros, (2018), el cual constaba de 50 ítems, pero para este estudio se adaptó debido a que la muestra incluía tres tipos de contextos diferentes. Al ser adaptado el FPSIS se realizó la validez de contenido por juicio de expertos, siendo cuatro expertos en el tema que residen en México, Panamá, Estados Unidos y Perú, quienes evaluaron el instrumento, sugiriendo realizar algunos cambios en la redacción y eliminar algunos ítems, lo cual se consideró hacerlo, como se puede observar en el anexo 2. La confiabilidad del cuestionario FPSIS se analizó con el Alfa de Cronbach (tabla 5) obteniendo $\alpha=0.916$ (Muy alta confiabilidad). El FPSIS, (tabla 3) posee 7 dimensiones y 44 ítems tipo escala de Likert, con una valoración de 0 (nunca) a 4 (siempre); autoaplicable en un tiempo aproximado de 25 minutos de administración colectiva.

Tabla 4.

Descripción del instrumento Cuestionario de Factores Psicosociales en el Trabajo Académico (FPSIS)

\begin{tabular}{llc}
\hline \multicolumn{1}{c}{ Dimensiones } & \multicolumn{1}{c}{ Ítems } & Instrumento \\
\hline Condiciones del lugar de trabajo & $1,2,3,4,5,6,7,8,9,10$ & \\
Carga de Trabajo & $11,12,13,14,15$ & Cuestionario de \\
Contenido y características de la tarea & $16,17,18,19,20,21,22,23$ & Factores \\
Exigencias laborales & $24,25,26,27,28,29$ & Psicosociales en \\
Papel de académico y desarrollo de la carrera & $30,31,32,33,34$ & el Trabajo \\
Interacción social y aspectos organizacionales & $35,36,37,38,39,40,41,42$ & Académico \\
Remuneración de rendimiento & 43,44 & (FPSIS)
\end{tabular}

Tabla 5.

Prueba de fiabilidad para la variable estrés docente y factores psicosociales

\begin{tabular}{ccc}
\hline Instrumento & Alfa de Cronbach & N $^{\circ}$ de elementos \\
\hline $\begin{array}{c}\text { Escala Docente ED-6 } \\
\text { Cuestionario de Factores } \\
\text { Psicosociales en el Trabajo } \\
\text { Académico (FPSIS) }\end{array}$ & 0.932 & 39 \\
\hline
\end{tabular}




\section{Tipo, diseño y enfoque del estudio}

El tipo de estudio es descriptivo, según Hernández, Fernández y Baptista (2014) los estudios descriptivos miden conceptos y definen variables, al describir contextos o fenómenos, en la que se busca especificar características y perfiles de los participantes, en este caso el estrés docente y los factores psicosociales. El diseño de estudio es no experimental, transversal y correlacional, en la que el investigador no manipula ninguna variable solo la mide a través de la aplicación de los instrumentos de recolección de datos en un solo momento y tiempo único, para contrastar si existe una relación entre ellas; en cuanto al enfoque es cuantitativo al haberse establecido mediciones, las cuales pasan por un proceso de análisis estadístico, (Hernández, Fernández \& Baptista, 2014).

\section{Procedimiento}

La ejecución de la investigación se realizó por etapas, como se describe a continuación: Primera etapa: Se realizó una revisión de la literatura para consolidar el problema detectado desde un fundamento teórico y que avalará el estudio a ejecutar, y a su vez permitiera poder elegir un instrumento validado en diversos países que formarán parte de la muestra, para hacer un diagnóstico sobre las variables de estudio. Segunda etapa: Se seleccionó la población a quien se le aplicaría los instrumentos para la recolección de datos, que todos los participantes fueran docentes en los diferentes niveles de educación, por lo menos con un aula asignada. Tercera etapa: Se publicó los dos instrumentos en una sola batería utilizando la plataforma de Google Forms, habiendo colocado en la primera sección el consentimiento informado, en el que se hizo énfasis la libertad de participar, y llenar los 2 instrumentos de recolección de datos de manera obligatoria. Cuarta etapa: Para la recolección de la información se envió la invitación para participar de la investigación a través del Whatsapp y el correo, en el que se incluyó un pequeño mensaje de presentación, donde se colocó el enlace de los cuestionarios, la administración de los cuestionarios se realizó en quince días consecutivos. Quinta etapa: Después de haber transcurrido el tiempo establecido se bajó el Consolidado del Excel que arroja el Google Forms, el mismo que se cuantifico para luego hacer el análisis descriptivo e inferencial mediante los programas de Excel y SPSS v.24.

\section{Resultados}

Se realizó el análisis inferencial a través de la prueba de Kruskal-Wallis, para poder contrastar la prueba de hipótesis general y las hipótesis especificas del estudio.

En la tabla 6 se puede observar el análisis de la relación entre el estrés docente y los factores psicosociales en general, en el que se contrasta una fuerte correlación positiva significativa entre el estrés y las condiciones del lugar de trabajo $(\mathrm{p}=0.000)$, carga de trabajo $(\mathrm{p}=0.003)$, contenido y características de la tarea $(\mathrm{p}=0.000)$, papel de académico y desarrollo de la carrera $(\mathrm{p}=0.001)$, interacción social y aspectos organizacionales $(\mathrm{p}=0.001)$; asimismo existe una fuerte correlación entre el estrés en general y los factores psicosociales en general $(\mathrm{p}=0.000)$, estos resultados concluyen que hay una relación entre el estrés docentes y los factores psicosociales en los docentes de Latinoamérica, Norteamérica y Europa. 
Tabla 6.

Resultados del análisis de correlación entre estrés docente y factores psicosociales

\begin{tabular}{lcc}
\hline & \multicolumn{2}{c}{ Nivel de estrés general } \\
\hline Factores psicosociales & Kruskal-Wallis & Sig. \\
\hline Condiciones del lugar de trabajo & 20.225 & $0.000^{*}$ \\
Carga de trabajo & 11.732 & $0.003^{*}$ \\
Contenido y características de la tarea & 18.055 & $0.000^{*}$ \\
Exigencias laborales & 1.635 & 0.441 \\
Papel de académico y desarrollo de la carrera & 14.134 & $0.001^{*}$ \\
& & \\
Interacción social y aspectos organizacionales & 14.009 & $0.001^{*}$ \\
Remuneración de rendimiento & & \\
Factores psicosociales en general & 5.714 & 0.057 \\
\end{tabular}

De la evaluación realizada en la tabla 7, se puede observar la relación entre el estrés y factores psicosociales en la dimensión condiciones del lugar de trabajo en docentes, de las que hay una relación directa y significativa con el nivel de estrés por ansiedad $(\mathrm{p}=0.001)$, nivel de estrés por depresión $(\mathrm{p}=0.003)$, nivel de estrés por creencias desadaptativas $(\mathrm{p}=0.015)$, nivel de estrés por presión laboral $(\mathrm{p}=0.000)$ y nivel de estrés por mal afrontamiento $(\mathrm{p}=0.030)$, por lo que se concluye que existe una relación significativa entre el estrés docente y factores psicosociales en la dimensión condiciones del lugar de trabajo en docentes de Latinoamérica, Norteamérica y Europa.

Tabla 7.

Análisis de la relación entre el estrés y factores psicosociales en la dimensión condiciones del lugar de trabajo en docentes.

\begin{tabular}{|c|c|c|c|c|c|c|c|c|c|c|}
\hline \multirow[b]{2}{*}{$\begin{array}{c}\text { Factores } \\
\text { psicosociale } \\
\text { s }\end{array}$} & \multicolumn{2}{|c|}{$\begin{array}{l}\text { Nivel de estrés } \\
\text { por ansiedad }\end{array}$} & \multicolumn{2}{|c|}{$\begin{array}{l}\text { Nivel de } \\
\text { estrés por } \\
\text { depresión }\end{array}$} & \multicolumn{2}{|c|}{$\begin{array}{l}\text { Nivel de estrés } \\
\text { por creencias } \\
\text { desadaptativas }\end{array}$} & \multicolumn{2}{|c|}{$\begin{array}{l}\text { Nivel de } \\
\text { estrés por } \\
\text { presión } \\
\text { laboral }\end{array}$} & \multicolumn{2}{|c|}{$\begin{array}{c}\text { Nivel de } \\
\text { estrés por mal } \\
\text { afrontamiento }\end{array}$} \\
\hline & $\begin{array}{c}\text { Krusk } \\
\text { al- } \\
\text { Wallis }\end{array}$ & Sig. & $\begin{array}{c}\text { Krusk } \\
\text { alWal } \\
\text { lis }\end{array}$ & Sig. & $\begin{array}{c}\text { Krusk } \\
\text { alWal } \\
\text { lis }\end{array}$ & Sig. & $\begin{array}{c}\text { Krusk } \\
\text { al- } \\
\text { Walli } \\
\text { s }\end{array}$ & Sig. & $\begin{array}{l}\text { Krusk } \\
\text { al- } \\
\text { Wallis }\end{array}$ & Sig. \\
\hline $\begin{array}{l}\text { Condiciones } \\
\text { del lugar de } \\
\text { trabajo }\end{array}$ & 13.773 & $\begin{array}{c}0.001 \\
*\end{array}$ & 11.482 & $\begin{array}{c}0.003 \\
*\end{array}$ & 8.412 & $0.015^{*}$ & 26.163 & $\begin{array}{c}0.000 \\
*\end{array}$ & 7.014 & $\begin{array}{c}0.030 \\
*\end{array}$ \\
\hline
\end{tabular}

De la evaluación realizada en la tabla 8 , se puede observar la relación entre el estrés y factores psicosociales en la dimensión carga de trabajo en docentes, de manera directa y significativa con el nivel de estrés por ansiedad $(\mathrm{p}=0.010)$, nivel de estrés por depresión $(\mathrm{p}=0.032)$, nivel de estrés por presión laboral $(\mathrm{p}=0.001)$, por lo que se concluye que existe una relación significativa entre el estrés docente en las dimensiones nivel de estrés por ansiedad, depresión y presión laboral y factores psicosociales en la dimensión carga de trabajo en docentes en docentes de Latinoamérica, Norteamérica y Europa Pero, no existe relación entre el estrés docente en las dimensiones nivel de estrés por creencias desadaptativas $(\mathrm{p}=0.303)$, y nivel de estrés por mal afrontamiento $(\mathrm{p}=0.193)$ con los factores psicosociales. 
Tabla 8.

Análisis de la relación entre el estrés y factores psicosociales en la dimensión carga de trabajo en docentes.

\begin{tabular}{|c|c|c|c|c|c|c|c|c|c|c|}
\hline \multirow[b]{2}{*}{$\begin{array}{l}\text { Factores } \\
\text { psicosocial } \\
\text { es }\end{array}$} & \multicolumn{2}{|c|}{$\begin{array}{c}\text { Nivel de estrés } \\
\text { por ansiedad }\end{array}$} & \multicolumn{2}{|c|}{$\begin{array}{l}\text { Nivel de estrés } \\
\text { por depresión }\end{array}$} & \multicolumn{2}{|c|}{$\begin{array}{c}\text { Nivel de } \\
\text { estrés por } \\
\text { creencias } \\
\text { desadaptativas }\end{array}$} & \multicolumn{2}{|c|}{$\begin{array}{c}\text { Nivel de estrés } \\
\text { por presión } \\
\text { laboral }\end{array}$} & \multicolumn{2}{|c|}{$\begin{array}{c}\text { Nivel de } \\
\text { estrés por mal } \\
\text { afrontamiento }\end{array}$} \\
\hline & $\begin{array}{c}\text { Krusk } \\
\text { al- } \\
\text { Walli } \\
\text { s }\end{array}$ & Sig. & $\begin{array}{l}\text { Krusk } \\
\text { al- } \\
\text { Wallis }\end{array}$ & Sig. & $\begin{array}{c}\text { Krusk } \\
\text { al- } \\
\text { Wallis }\end{array}$ & Sig. & $\begin{array}{l}\text { Krusk } \\
\text { al- } \\
\text { Wallis }\end{array}$ & Sig. & $\begin{array}{c}\text { Krusk } \\
\text { al- } \\
\text { Wallis }\end{array}$ & Sig. \\
\hline $\begin{array}{c}\text { Carga de } \\
\text { trabajo }\end{array}$ & 9.249 & $0.010 *$ & 6.855 & $0.032 *$ & 2.386 & 0.303 & 14.379 & $0.001 *$ & 3.287 & 0.193 \\
\hline
\end{tabular}

De la evaluación realizada en la Tabla 9, se puede observar una relación significativa positiva entre el estrés y factores psicosociales en la dimensión contenido y característica de la tarea en docentes, con el nivel de estrés por ansiedad $(\mathrm{p}=0.008)$, nivel de estrés por depresión $(\mathrm{p}=0.026)$, nivel de estrés por creencias desadaptativas $(\mathrm{p}=0.006)$, nivel de estrés por presión laboral ( $\mathrm{p}=0.000$ ), por lo que se concluye que se correlacionan significativamente el estrés docente y los factores psicosociales en la dimensión contenido y características de la tarea en docentes de Latinoamérica, Norteamérica y Europa. Pero no se encontró relación entre el estrés por mal afrontamiento y los factores psicosociales en la dimensión contenido y característica de la tarea.

Tabla 9.

Análisis de la relación entre el estrés y factores psicosociales en la dimensión contenido y característica de la tarea en docentes

\begin{tabular}{ccccccccccc}
\hline & $\begin{array}{c}\text { Nivel de estrés } \\
\text { por ansiedad }\end{array}$ & \multicolumn{2}{c}{$\begin{array}{c}\text { Nivel de estrés } \\
\text { por depresión }\end{array}$} & \multicolumn{2}{c}{$\begin{array}{c}\text { Nivel de estrés } \\
\text { por creencias } \\
\text { desadaptativas }\end{array}$} & $\begin{array}{c}\text { Nivel de estrés } \\
\text { por presión } \\
\text { laboral }\end{array}$ & $\begin{array}{c}\text { Nivel de estrés } \\
\text { por mal } \\
\text { afrontamiento }\end{array}$ \\
\hline $\begin{array}{c}\text { Factores } \\
\text { psicosociale } \\
\text { s }\end{array}$ & $\begin{array}{c}\text { Kruskal } \\
\text {-Wallis }\end{array}$ & Sig. & $\begin{array}{l}\text { Kruskal } \\
\text {-Wallis }\end{array}$ & Sig. & $\begin{array}{c}\text { Kruskal } \\
\text {-Wallis }\end{array}$ & Sig. & $\begin{array}{c}\text { Kruskal } \\
\text {-Wallis }\end{array}$ & Sig. & $\begin{array}{c}\text { Kruskal } \\
\text {-Wallis }\end{array}$ & Sig. \\
\hline $\begin{array}{c}\text { Contenido y } \\
\text { característic } \\
\text { as de la tarea }\end{array}$ & 9.709 & 0.008 & 7.336 & 0.026 & 10.339 & 0.006 & 21.915 & 0.000 & 2.830 & 0.24 \\
& & $*$ & & $*$ & & $*$ & & & & \\
\hline
\end{tabular}

De la evaluación realizada en la Tabla 10, en la cual se analiza la relación entre estrés y factores psicosociales en la dimensión exigencias laborales en docentes, se observa que no existe correlación entre estas variables y sus dimensiones.

Tabla 10.

Análisis de la relación entre el estrés y factores psicosociales en la dimensión exigencias laborales en docentes.

\begin{tabular}{cccccccccc}
\hline & $\begin{array}{c}\text { Nivel de estrés } \\
\text { por ansiedad }\end{array}$ & \multicolumn{2}{c}{$\begin{array}{c}\text { Nivel de estrés } \\
\text { por depresión }\end{array}$} & $\begin{array}{c}\text { Nivel de estrés } \\
\text { por creencias } \\
\text { desadaptativas }\end{array}$ & $\begin{array}{c}\text { Nivel de estrés } \\
\text { por presión } \\
\text { laboral }\end{array}$ & $\begin{array}{c}\text { Nivel de } \\
\text { estrés por mal } \\
\text { afrontamiento }\end{array}$ \\
\hline $\begin{array}{c}\text { Factores } \\
\text { psicosoci } \\
\text { ales }\end{array}$ & $\begin{array}{c}\text { Kruskal- } \\
\text { Wallis }\end{array}$ & Sig. & $\begin{array}{c}\text { Kruskal } \\
\text {-Wallis }\end{array}$ & Sig. & $\begin{array}{c}\text { Kruskal } \\
\text {-Wallis }\end{array}$ & Sig. & $\begin{array}{c}\text { Kruskal } \\
\text {-Wallis }\end{array}$ & $\begin{array}{c}\text { Sig. } \\
\text { Krusk } \\
\text { al- }\end{array}$ & Sig. \\
Walli \\
Exigenci \\
$\begin{array}{c}\text { as } \\
\text { laborales }\end{array}$
\end{tabular}


De la evaluación realizada en la Tabla 10, en la cual se analiza la relación entre el estrés y factores psicosociales en dimensión papel del académico y desarrollo de la carrera en docentes, se observa que las condiciones del nivel de estrés por ansiedad $(\mathrm{p}=0.001)$, nivel de estrés por depresión $(\mathrm{p}=0.001)$, nivel de estrés por presión laboral $(\mathrm{p}=0.000)$ y nivel de estrés por mal afrontamiento ( $\mathrm{p}=0.001)$ se correlacionan significativamente, por lo que se concluye que el estrés se relaciona con los factores psicosociales en la dimensión el papel de académico y desarrollo de la carrera en los docentes de Latinoamérica, Norteamérica y Europa.

Tabla 11.

Análisis de la relación entre el estrés y factores psicosociales en dimensión papel del académico $y$ desarrollo de la carrera en docentes.

\begin{tabular}{ccccccccccc}
\hline & \multicolumn{2}{c}{$\begin{array}{c}\text { Nivel de estrés } \\
\text { por ansiedad }\end{array}$} & \multicolumn{2}{c}{$\begin{array}{c}\text { Nivel de estrés } \\
\text { por depresión }\end{array}$} & \multicolumn{2}{c}{$\begin{array}{c}\text { Nivel de estrés } \\
\text { por creencias } \\
\text { desadaptativas }\end{array}$} & $\begin{array}{c}\text { Nivel de estrés } \\
\text { por presión } \\
\text { laboral }\end{array}$ & \multicolumn{2}{c}{$\begin{array}{c}\text { Nivel de estrés } \\
\text { por mal } \\
\text { afrontamiento }\end{array}$} \\
\hline $\begin{array}{c}\text { Factores } \\
\text { psicosociale } \\
\text { s }\end{array}$ & $\begin{array}{l}\text { Kruskal } \\
\text {-Wallis }\end{array}$ & Sig. & $\begin{array}{l}\text { Kruskal } \\
\text {-Wallis }\end{array}$ & Sig. & $\begin{array}{c}\text { Kruskal } \\
\text {-Wallis }\end{array}$ & Sig. & $\begin{array}{c}\text { Kruskal } \\
\text {-Wallis }\end{array}$ & Sig. & $\begin{array}{c}\text { Kruskal } \\
\text {-Wallis }\end{array}$ & Sig. \\
\hline $\begin{array}{c}\text { Papel de } \\
\text { académico y } \\
\text { desarrollo }\end{array}$ & 14.877 & 0.001 & 13.417 & 0.001 & 3.196 & 0.20 & 17.484 & 0.000 & 9.170 & 0.001 \\
de la carrera & & $*$ & & $*$ & & 2 & & $*$ & & $*$ \\
\hline
\end{tabular}

De la evaluación realizada en la Tabla 12, en la cual se analiza la relación entre el estrés y factores psicosociales en la dimensión interacción social y aspectos organizacionales en docentes, se observa que las condiciones del nivel de estrés por ansiedad ( $\mathrm{p}=0.003)$, nivel de estrés por depresión $(\mathrm{p}=0.017)$, nivel de estrés por creencias desadaptativa $(\mathrm{p}=0.005)$, nivel de estrés por presión laboral $(\mathrm{p}=0.000)$ y nivel de estrés por mal afrontamiento $(\mathrm{p}=0.000)$ se correlacionan significativamente con la interacción social y aspectos organizacionales. Se concluye que existe una relación significativa positiva entre el estrés y los factores psicosociales en la dimensión interacción social y aspectos organizaciones en docentes de Latinoamérica, Norteamérica y Europa.

Tabla 12.

Análisis de la relación entre el estrés y factores psicosociales en la dimensión interacción social y aspectos organizacionales en docentes.

\begin{tabular}{|c|c|c|c|c|c|c|c|c|c|c|}
\hline \multirow[b]{2}{*}{$\begin{array}{c}\text { Factores } \\
\text { psicosocia } \\
\text { les }\end{array}$} & \multicolumn{2}{|c|}{$\begin{array}{c}\text { Nivel de estrés } \\
\text { por ansiedad }\end{array}$} & \multicolumn{2}{|c|}{$\begin{array}{l}\text { Nivel de estrés } \\
\text { por depresión }\end{array}$} & \multicolumn{2}{|c|}{$\begin{array}{l}\text { Nivel de estrés } \\
\text { por creencias } \\
\text { desadaptativas }\end{array}$} & \multicolumn{2}{|c|}{$\begin{array}{l}\text { Nivel de } \\
\text { estrés por } \\
\text { presión } \\
\text { laboral }\end{array}$} & \multicolumn{2}{|c|}{$\begin{array}{l}\text { Nivel de estrés } \\
\text { por mal } \\
\text { afrontamiento }\end{array}$} \\
\hline & $\begin{array}{c}\text { Krusk } \\
\text { al- } \\
\text { Wallis }\end{array}$ & Sig. & $\begin{array}{l}\text { Krus } \\
\text { kal- } \\
\text { Walli } \\
\text { s }\end{array}$ & Sig. & $\begin{array}{l}\text { Krusk } \\
\text { al- } \\
\text { Wallis }\end{array}$ & Sig. & $\begin{array}{c}\text { Krus } \\
\text { kal- } \\
\text { Wall } \\
\text { is }\end{array}$ & Sig. & $\begin{array}{c}\text { Krusk } \\
\text { al- } \\
\text { Wallis }\end{array}$ & Sig. \\
\hline $\begin{array}{l}\text { Interacció } \\
\mathrm{n} \text { social y } \\
\text { aspectos } \\
\text { organizaci } \\
\text { onales }\end{array}$ & $\begin{array}{c}11.61 \\
3\end{array}$ & $0.003 *$ & 8.202 & $\begin{array}{c}0.017 \\
*\end{array}$ & $\begin{array}{c}10.57 \\
0\end{array}$ & $0.005^{*}$ & $\begin{array}{c}18.6 \\
49\end{array}$ & $\begin{array}{c}0.00 \\
0^{*}\end{array}$ & 15.437 & $\begin{array}{c}0.00 \\
0 *\end{array}$ \\
\hline
\end{tabular}

De la evaluación realizada en la Tabla 13, en la cual se analiza la relación entre el estrés y factores psicosociales en la dimensión remuneración de rendimiento en docentes, se observa que el nivel de estrés por presión laboral $(\mathrm{p}=0.025)$ se correlaciona con la dimensión remuneración del rendimiento. No encontrándose relación entre nivel de estrés por ansiedad, depresión, por creencias desadaptativas y por mal afrontamiento no se correlacional con los factores psicosociales en la dimensión remuneración del rendimiento. 
Tabla 13.

Análisis de la relación entre el estrés y factores psicosociales en la dimensión remuneración de rendimiento en docentes.

\begin{tabular}{|c|c|c|c|c|c|c|c|c|c|c|}
\hline \multirow[b]{2}{*}{$\begin{array}{c}\text { Factores } \\
\text { psicosociale } \\
\text { s } \\
\end{array}$} & \multicolumn{2}{|c|}{$\begin{array}{l}\text { Nivel de estrés } \\
\text { por ansiedad }\end{array}$} & \multicolumn{2}{|c|}{$\begin{array}{l}\text { Nivel de estrés } \\
\text { por depresión }\end{array}$} & \multicolumn{2}{|c|}{$\begin{array}{l}\text { Nivel de estrés } \\
\text { por creencias } \\
\text { desadaptativas }\end{array}$} & \multicolumn{2}{|c|}{$\begin{array}{l}\text { Nivel de estrés } \\
\text { por presión } \\
\text { laboral }\end{array}$} & \multicolumn{2}{|c|}{$\begin{array}{l}\text { Nivel de estrés } \\
\text { por mal } \\
\text { afrontamiento }\end{array}$} \\
\hline & $\begin{array}{c}\text { Kruska } \\
1- \\
\text { Wallis }\end{array}$ & Sig. & $\begin{array}{c}\text { Kruska } \\
1- \\
\text { Wallis }\end{array}$ & Sig. & $\begin{array}{c}\text { Kruska } \\
1- \\
\text { Wallis }\end{array}$ & Sig. & $\begin{array}{c}\text { Kruska } \\
1- \\
\text { Wallis }\end{array}$ & Sig. & $\begin{array}{c}\text { Kruska } \\
1- \\
\text { Wallis }\end{array}$ & Sig. \\
\hline $\begin{array}{l}\text { Remuneraci } \\
\text { ón del } \\
\text { rendimiento }\end{array}$ & 1.665 & $\begin{array}{c}0.43 \\
5\end{array}$ & 2.160 & $\begin{array}{c}0.34 \\
0\end{array}$ & 3.151 & $\begin{array}{c}0.20 \\
7\end{array}$ & 7.388 & $\begin{array}{c}0.025 \\
*\end{array}$ & 0.872 & $\begin{array}{c}0.64 \\
7\end{array}$ \\
\hline
\end{tabular}

\section{Discusión}

De acuerdo con los resultados obtenidos del contraste de la hipótesis general, existe relación positiva significativa $(p=0.000)$ entre el estrés y los factores psicosociales en los docentes, lo cual coincide con Graneros, (2018); Donayre (2016) al referir que aspectos relacionados al ámbito laboral como el entorno físico generan estrés en los docentes lo que puede derivar en un estrés más agudo denominado burnout, por su parte Orozco, (2018); Chavarría et al., (2017); Osorio y Cárdenas, (2016) refieren que el estrés es una afectación mental que es influenciada por las demandas físico emocionales producida por la interacción con los estudiantes de manera negativa aunada a los problemas de los factores psicosociales, lo que dificulta el desempeño docente. Chiang, Heredia y Santamaría, (2017) que el estrés es un factor que se presenta por las exigencias laborales en el puesto de trabajo asociada a la escasez de recursos físicos, materiales y el interactuar en ambientes de tensión. Díaz, (2014) en su investigación determinó que los docentes que padecen estrés están propensos a desarrollar burnout por los constantes factores de riesgo psicosocial que se les presenta, enfatizando la convivencia a diario que tienen con los estudiantes. Iniesta (2016) enfatiza que en Europa el estrés laboral afecta a casi 40 millones de trabajadores produciendo efectos nocivos en la salud de estos y la productividad y competitividad de las empresas, haciéndose necesaria una inversión necesaria de 20,000 millones de euros al año para combatirlo.

Estos resultados aseveran que los estados del ánimo como la depresión y ansiedad están vinculados con el estrés; según García-Rivera, Maldonado-Radillo y Ramírez, (2014) que tanto la depresión como la ansiedad generan alteraciones del humor manifestados con irritabilidad, falta de capacidad de sentir placer, apetito, insomnio, para Bermúdez, (2018) la depresión es un afectación mental que lo padece aproximadamente 350 millones de personas en el mundo, considerada como una de las discapacidades que influye en el absentismo laboral, al estar unida al estrés, a su vez incide en la disminución del rendimiento y productividad del trabajador, de acuerdo a Iniesta (2016); Fernández-Puig, (2015). Martínez, (2015) hace mención que la relación que pueda existir entre el individuo y la valoración que este utilice como estrategia de afrontamiento si estas son las adecuadas y suficientes permitirá que se adapte a estas situaciones estresantes o amenazadora para que no le genere estrés. Chiang, Heredia y Santamaría, (2017) mencionan que el nivel de estrés por presión laboral y la ansiedad son factores que afectan al trabajador al estar ligadas con las exigencias laborales lo que les crea un desajuste en su salud física y psicológica con cambios psicosomáticos perjudicando su desempeño profesional.

Cardozo, (2018) demanda que se generen políticas de intervención multidimensional en la comunidad docente con la finalidad de paliar esta situación problemática y brindar una mejor calidad laboral para la mejora en el desempeño y compromiso de la labor docente. Lemos et al., (2018) en su investigación realizada sobre factores psicosociales asociados al estrés en profesores universitarios colombianos coincide con este estudio al referir que las desbordantes exigencias en el trabajo académico, la variabilidad y sobrecarga en las tareas son factores de riesgo para la salud 
física y psicológica del docente, encontrando que hay una presencia alta de niveles de estrés, depresión y ansiedad significativos en los docentes que formaron parte de la investigación. Castillo, Fernández y López, (2014) refieren que los docentes experimentan depresión y ansiedad por el rol académico que cumplen con toda la comunidad estudiantil por estar expuestos a estresores agudos de diversa índole. Vargas, (2018); Chiang, Heredia y Santamaría, (2017) enfatizan que el estrés laboral tiene una incidencia en los trabajadores en cuanto a los factores psicosociales relacionados a la exigencia y presión laboral, complejidad de la tarea, sobre carga de trabajo, actividades rutinarias, falta de apoyo en el desarrollo profesional del trabajador. Domínguez-Rodríguez, Prieto-Cabras y Barraca-Mairal, (2017) la sobrecarga de trabajo dentro de las actividades asignadas o la realización de tareas que no le corresponden o tareas repetitivas diariamente por tiempos prolongado o tareas que no le competen conllevan a un cuadro de estrés, ansiedad y depresión al trabajador, lo mismo que los trabajos administrativos y cambios de roles a que está sometido el docente (Chavarría et al., 2017; Donayre, 2016).

Para Vargas, (2018); Vieco y Abello, (2014) la afectación que tiene los factores psicosociales como se muestran en estos resultados llevan a consecuencias negativas para la institución ya que influye en el ausentismo del trabajador a su puesto de labores, sea esta por causas justificadas o injustificadas, incrementando horas de trabajo perdidas para la organización, lo que recae en una baja productividad, mala calidad del servicio y una sobrecarga de trabajo en los demás trabajadores. Asimismo, Vargas, (2018) que la implicancia de las interacciones sociales en el trabajo puede convertirse en un incentivo para un mejor desempeño del trabajador, ya que a través de esta se construyen relaciones sociales al interior de la organización dando sentido de pertinencia a estos, pero en este estudio se ha podido concluir que existe una interferencia con esta dimensión por estar correlacionado con ansiedad, depresión, creencias desadaptativas, mal afrontamiento y el estrés. Cháidez y Barraza, (2018); Solanes, et al., (2013) hacen mención que el afrontamiento al estrés en docentes, es un esfuerzo cognitivo y conductual para resolver demandas internas y/o externas especificas que son excesivas para las personas y ponen en peligro su bienestar, lo que coincide en este estudio al estar presente el mal afrontamiento al estrés en casi la mayoría de las correlaciones con los factores psicosociales.

Donayre, (2016) refiere que adicional a unas buenas relaciones laborales y de comunicación efectiva con todos los miembros de la comunidad educativa, la cual favorece la valoración positiva de la labor realizada aunada a un reconocimiento monetario e incentivos económicos permiten que los docentes sientan un bienestar óptimo, pero si este es todo lo contrario lo percibirán como factores estresantes, aspecto que coincide con los resultados obtenidos en este estudio.

Para futuros estudios, sería importante incluir una mayor cantidad de muestra de docentes en estudios de este tipo donde se incluyen diversos países de diferentes continentes, ya que en esta investigación es una limitante, al no poder aseverar si todos los resultados pueden generalizarse, aunque hubo resultados interesantes adiciones relacionados a otros aspectos sociodemográficos y generales, que sería relevante se amplié dentro de la temática de estrés docente y factores psicosociales.

\section{Referencias}

Barradas, M., Trujillo, P., Guzmán, M., \& López, J., (2015). Nivel de estrés en docentes universitarios. Revista Iberoamericana de Contaduría, Economía y Administración, 4(8), 1-21. doi: https://doi.org/10.23913/ricea.v4i8.38

Bermúdez, V. (2018). Ansiedad, depresión, estrés y autoestima en la adolescencia. Relación, implicaciones y consecuencias en la educación privada. Cuestiones Pedagógicas, 26, $37-$ 52. doi: http://dx.doi.org/10.12795/CP.2017.i26.03

Castillo, A., Fernández, R., \& López, P. (2014). Prevalencia de ansiedad y depresión en docentes. Enfermería del trabajo, 4, 55-62. 
Castro, P. (2008). El estrés docente en los profesores de escuela pública (Tesis de Maestría). Pontificia Universidad Católica del Perú, Perú.

Cardozo, L., (2016). El estrés en el profesorado. Reflexiones en psicología, 15, 75-98.

Cháidez, J., \& Barraza, A., (2018). Afrontamiento al estrés y su relación con el tipo de jornada laboral en docentes de educación primaria. Informes Psicológicos, 18(2), 63-75. doi: https://doi.org/10.18566/nfpsic.v18n2a04

Chavarría, R., Colunga, F., Loria, J. \& Peláez, K. (2017). Síndrome de burnout en medico docentes de un hospital de 2o nivel en México. Educación Médica, 18(4), 254-261. doi: https://doi.org/10.1016/j.edumed.2016.09.001

Chiang, M., Heredia, S., \& Santamaría, E., (2017). Clima organizacional y salud psicológica: una dualidad organizacional. Dimensión Empresarial, 15(1), 63-76. doi: https://doi.org/10.15665/rde.v15i1.641

Chiang, M., Riquelme, G., \& Rivas, P. (2018). Relación entre satisfacción laboral, estrés laboral y sus resultados en trabajadores de una institución de beneficencia de la provincia de Concepción. Ciencia \& Trabajo, 20(63), 178-186. doi: https://doi.org/10.4067/S0718-24492018000300178

De la Cruz, E. (2017). Estrés laboral y desempeño docente en la I.E. No. 89002, Chimbote, 2017 (Tesis de Maestría). Universidad César Vallejo, Perú.

Díaz, A. (2014). Influencia del síndrome de burnout en el desempeño docente de los profesores de la facultad de ciencias de la salud de la Universidad Nacional del Callao-2013 (Tesis de doctorado). Universidad Nacional Mayor de San Marcos, Perú.

Díaz, D. (2011). Estrés laboral y sus factores de riesgo psicosocial. Revista CES Salud Publica, 2(1), 80-84.

Domínguez-Rodríguez, I., Prieto-Cabras, V. \& Barraca-Mairal, J. (2017). Un estudio de caso de trastorno adaptativo con ansiedad por situación de sobrecarga laboral. Clínica y Salud, 28, 139-146. doi: https://doi.org/10.1016/j.clysa.2017.05.003

Donayre, C. (2016). Percepción sobre estrés laboral en docentes de educación básica regular de nivel secundario que aplican programas internacionales en una institución educativa privada bilingüe (Tesis de Maestría). Pontificia Universidad Católica del Perú, Perú.

Espadas, P., (2016). Factores psicosociales de riesgo: Estrés laboral y Burnout (Tesis de Pregrado). Universitat de les Illes Balears, España.

Fernández-Puig, Longás, J., Chamarro, A. \& Virgili, C., (2015). Evaluando la salud laboral de los docentes de centros concertados: el cuestionario de salud docente. Journal of Wrok and Organizational Psychology, 31, 175-185. doi: https://doi.org/10.1016/j.rpto.2015.07.001

García-Moran, M., \& Gil-Lacruz, M., (2016). El estrés en el ámbito de los profesionales de la salud. Persona 19, 11-30. doi: https://doi.org/10.26439/persona2016.n019.968

García-Rivera, B., Maldonado-Radillo, S., \& Ramírez, M. (2014); Estados afectivos emocionales (depresión, ansiedad y estrés) en personal de enfermería del sector salud pública de México. Summa $\quad$ Psicológica, $11(1), \quad 65-73 . \quad$ doi: https://doi.org/10.18774/448x.2014.11.128

González, Ch., (2018). Estrés laboral en docentes de la primera infancia (Tesis de pregrado). Corporación Universitaria Minuto de Dios. Bogotá, Colombia.

Graneros, J. (2018). Factores psicosociales en el trabajo y estrés laboral en colaboradores de una empresa de ingeniería-Lima, 2017 (Tesis de Maestría). Universidad Peruana Cayetano Heredia, Perú.

Hernández, Fernández \& Baptista, (2014). Metodología de la Investigación (6ta. Ed.) México: McGraw-Hill.

Iniesta, A. (2016). Guía sobre el manejo del estrés desde medicina del trabajo. Edición: Sans Growing Brands, Barcelona, España. 
Lemos, M., Calle, G., Roldán, T., Valencia, M., Orejuela, J., \& Román-Calderón, J., (2018). Factores psicosociales asociados al estrés en profesores universitarios colombianos. doi: https://doi.org/10.15332/s1794-9998.2019.0001.05

Martínez, J., (2015). Cómo se defiende el profesorado de secundaria del estrés: burnout y estrategias de afrontamiento. Journal of Wrok and Organizational Psychology, 31, 1-9. doi: https://doi.org/10.1016/j.rpto.2015.02.001

Montiel-Jarquín, Á., Torres-Castillo, M., Herrera-Velasco, M. Ahuamada-Sánchez, Ó., Barragán-Hervella, R., García-Villaseñor, A., \& Loría-Castellanos, J., (2015). Estado actual de depresión y ansiedad en residentes de traumatología y ortopedia en una unidad de tercer nivel de atención médica. Educación Médica, 16(2), 116-125. doi: https://doi.org/10.1016/j.edumed.2015.09.006

Orozco, J. (2018). Nivel de estrés de los docentes de la facultad de humanidades de psicología clínica y psicología industrial/organizacional de dedicación completa de la Universidad Rafael Landivar (Tesis de pregrado). Universidad Rafael Landívar, Guatemala.

Osorio, J., \& Cárdenas, L., (2017). Estrés laboral: estudio de revisión. Revista Diversitas Perspectivas en Psicología, 13(1), 81-90. doi: https://doi.org/10.15332/s17949998.2017.0001.07

Patiño, A. \& Gutiérrez, H. (2013). Análisis de factores psicosociales asociados al estrés laboral en docentes de planta de las facultades de ciencias económicas administrativas e ingenierías de la Universidad Autónoma de Occidente (Tesis de pregrado). Universidad Autónoma de Occidente, Colombia.

Parihuaman-Aniceto, M. (2017) Nivel de estrés de los docentes de las instituciones educativas de Villa Vicús y Kilómetro 50, distrito de Chulucanas-Morropón-Piura (Tesis de Maestría). Universidad de Piura, Perú.

Perez, J. (2018). Evaluación de factores de riesgo psicosocial y estrés laboral de los docentes de la facultad de ingeniería y arquitectura de la Universidad Peruana Unión Filial Tarapoto, 2017 (Tesis de Maestría). Universidad Peruana Unión, Perú.

Sánchez, R., (2017). Factores sociolaborales y estrés en docentes de secundaria de la ciudad de Huancayo. (Tesis de Maestría) Universidad Nacional del Centro del Perú.

Solanes, A., Del Río, B., Rodríguez, K., Benavides, G., \& Martínez, F. (2013). Factores de riesgo psicosocial laboral y burnout en función de la categoría profesional. Revista de Psicología de la Salud, 11(1), 45-63. doi: https://doi.org/10.21134/pssa.v1i1.373

Vargas, M. (2018). Diagnóstico de factores de riesgos psicosociales y propuesta de un plan de mejora en la empresa Franco Granda Dimalvid Cia. Ltda. (Tesis de pregrado). Universidad de Central del Ecuador, Ecuador.

Vieco, G. \& Abello, R., (2014). Factores psicosociales de origen laboral, estrés y morbilidad en el mundo. Psicología desde el caribe, 31(2), 354-385. doi: https://doi.org/10.14482/psdc.31.2.5544

Villanueva, M., Jiménez, I., García, L., \& Durán, J. (2005). Valoración de las fuentes de estrés laboral en personal docente. Servicio de Higiene Industrial y Salud Laboral. Consejería de Trabajo y Política Social de la Región de Murcia, España.

Unda, S., Uribe, F., Jurado, S., García, M., Tovalín, H., \& Juárez, A. (2016). Elaboración de una escala para valorar los factores de riesgo psicosocial en el trabajo de profesores universitarios. Journal of Wrok and Organizational Psychology, 32, 67-74. doi: https://doi.org/10.1016/j.rpto.2016.04.004

Zuñiga-Jara, S. \& Pizarro-León, V. (2018). Mediciones de estrés laboral en docentes de un colegio público regional chileno. Información Tecnológica, 29(1), 171-180. doi: https://doi.org/10.4067/S0718-07642018000100171 
A continuación, va a encontrar una serie de afirmaciones, de las cuales deberá elegir la que a su parecer le corresponde, considerando una valoración de 1 a 5 , tomando en cuenta que:

$1=$ totalmente de acuerdo $2=$ de acuerdo $\quad 3=$ indiferente, ni uno ni el otro

$4=$ en desacuerdo $\quad 5=$ totalmente en desacuerdo

\begin{tabular}{|c|c|c|c|c|c|c|}
\hline \multirow{2}{*}{ No. } & Ítem & & & & & \\
\hline & Nivel de estrés por ansiedad & 1 & 2 & 3 & 4 & 5 \\
\hline 1 & Me cuesta tranquilizarme tras los contratiempos laborales & & & & & \\
\hline 2 & $\begin{array}{l}\text { En muchos momentos de la jornada laboral me siento tenso y } \\
\text { nervioso }\end{array}$ & & & & & \\
\hline 3 & La tensión del trabajo está alterando mis hábitos de sueño & & & & & \\
\hline 4 & Hay tareas laborales que afronto con temor. & & & & & \\
\hline 5 & Los problemas laborales están afectando mi salud física & & & & & \\
\hline 6 & La tensión en el trabajo está alterando mis hábitos alimenticios & & & & & \\
\hline 7 & $\begin{array}{l}\text { En la Institución Educativa se dan situaciones de tensión que } \\
\text { hacen que me entre sudores fríos. }\end{array}$ & & & & & \\
\hline 8 & $\begin{array}{l}\text { Ante los problemas en el trabajo siento que se me altera la } \\
\text { respiración }\end{array}$ & & & & & \\
\hline 9 & $\begin{array}{l}\text { La tensión laboral hace que vaya al baño con más frecuencia de } \\
\text { lo normal }\end{array}$ & & & & & \\
\hline \multirow[t]{2}{*}{10} & $\begin{array}{l}\text { Tomo algunos tranquilizantes o fármacos para aliviar mi } \\
\text { malestar físico por sentir presión en el trabajo. }\end{array}$ & & & & & \\
\hline & Nivel de estrés por depresión & & & & & \\
\hline \multirow[t]{2}{*}{11} & $\begin{array}{l}\text { Me siento triste con más frecuencia de lo que era normal en mí } \\
\text { por los problemas laborales }\end{array}$ & & & & & \\
\hline & ELIMINO & & & & & \\
\hline 12 & Tiendo a ser pesimista ante los problemas del trabajo & & & & & \\
\hline 13 & Veo el futuro sin ilusión alguna. & & & & & \\
\hline 14 & Tengo la sensación de estar desmoronándome emocionalmente. & & & & & \\
\hline 15 & Siento ganas de llorar sin una razón aparente & & & & & \\
\hline 16 & Me falta energía para afrontar la labor de profesor. & & & & & \\
\hline \multirow[t]{2}{*}{17} & Me cuesta concentrarme cuando me pongo a trabajar. & & & & & \\
\hline & Nivel de estrés por creencias desadaptativas & & & & & \\
\hline 18 & $\begin{array}{l}\text { Creo que no hay buenos o malos profesores, sino buenos o } \\
\text { malos alumnos. }\end{array}$ & & & & & \\
\hline 19 & $\begin{array}{l}\text { Incluir alumnos con Necesidades Educativas Especiales en el } \\
\text { aula es un error que perjudica el rendimiento del resto }\end{array}$ & & & & & \\
\hline 20 & Me pagan por enseñar, no por formar personas. & & & & & \\
\hline \multirow[t]{2}{*}{21} & $\begin{array}{l}\text { Me incómoda tener que enseñar a estudiantes que no valoran la } \\
\text { educación }\end{array}$ & & & & & \\
\hline & Nivel de estrés por presión laboral & & & & & \\
\hline 22 & Acabo las jornadas de trabajo extenuado/a. & & & & & \\
\hline 23 & $\begin{array}{l}\text { A medida que avanza la jornada laboral siento más necesidad de } \\
\text { que ésta acabe }\end{array}$ & & & & & \\
\hline 24 & Realizar mis programaciones curriculares me resulta difícil & & & & & \\
\hline 25 & $\begin{array}{l}\text { A algunos alumnos lo único que les pido es que no me molesten } \\
\text { mientras enseño a los demás. }\end{array}$ & & & & & \\
\hline 26 & $\begin{array}{l}\text { Hay clases en las que empleo más tiempo en llamar la atención } \\
\text { (reñir) que en explicar. }\end{array}$ & & & & & \\
\hline 27 & $\begin{array}{l}\text { Los malos momentos personales de los estudiantes me afectan } \\
\text { personalmente }\end{array}$ & & & & & \\
\hline 28 & Me afecta las agresiones verbales por parte de los estudiantes & & & & & \\
\hline
\end{tabular}




\begin{tabular}{|l|l|l|l|l|l|}
\hline 29 & $\begin{array}{l}\text { Me desmotiva la falta de apoyo de los padres en problemas de } \\
\text { disciplina de sus hijos }\end{array}$ & & & \\
\hline 30 & Me afecta la rivalidad entre grupos de profesores & & & & \\
\hline 31 & Siento estar lejos de mi autorrealización profesional y laboral & & & & \\
\hline 32 & $\begin{array}{l}\text { Estoy bastante distanciado del ideal de profesor con el que } \\
\text { comencé }\end{array}$ & & & \\
\hline 33 & He perdido la motivación por la enseñanza. & & & & \\
\hline 34 & $\begin{array}{l}\text { Me desalienta la inestabilidad de mi puesto como docente en la } \\
\text { institución educativa }\end{array}$ & & & & \\
\hline 35 & Nivel de estrés por mal afrontamiento & & & & \\
\hline 36 & $\begin{array}{l}\text { Mis relaciones sociales fuera de la institución (familia, pareja, } \\
\text { amigos, etc.) son muy buenas. }\end{array}$ & & & & \\
\hline 37 & Mis compañeros cuentan con mi apoyo moral y profesional. & & & & \\
\hline 38 & $\begin{array}{l}\text { Afronto con eficacia los problemas que a veces surgen con los } \\
\text { compañeros de trabajo. }\end{array}$ & & & \\
\hline 39 & $\begin{array}{l}\text { Resuelvo con facilidad los problemas del trabajo y con mis } \\
\text { estudiantes. }\end{array}$ & & & \\
\hline
\end{tabular}

Anexo 2

Cuestionario Factores Psicosociales en el Trabajo Académico (FPSIS)

A continuación, va a encontrar una serie de afirmaciones acerca de las cuales deberá marcar una de las opciones siguientes:

$0=$ nunca $\quad 1=$ casi nunca $\quad 2=$ algunas veces $3=$ casi siempre $\quad 4=$ siempre

\begin{tabular}{|c|c|c|c|c|c|c|}
\hline No. & Condiciones del lugar de trabajo & 0 & 1 & 2 & 3 & 4 \\
\hline 1 & El ruido en general interfiere en mis actividades académicas & & & & & \\
\hline 2 & $\begin{array}{l}\text { La iluminación de mi aula no es la adecuada en la institución } \\
\text { educativa }\end{array}$ & & & & & \\
\hline 3 & $\begin{array}{l}\text { La temperatura en el aula y/u otros ambientes académicos no es } \\
\text { adecuada }\end{array}$ & & & & & \\
\hline 4 & $\begin{array}{l}\text { Existe mala higiene en mi aula y otros ambientes de la } \\
\text { institución educativa }\end{array}$ & & & & & \\
\hline 5 & $\begin{array}{l}\text { Está expuesto (a) a polvos, gases, solventes o vapores en el aula } \\
\text { donde imparte sus clases }\end{array}$ & & & & & \\
\hline 6 & $\begin{array}{l}\text { Está expuesto (a) a microbios, hongos, insectos o roedores en el } \\
\text { aula donde imparte sus clases }\end{array}$ & & & & & \\
\hline 7 & Carece de una sala de profesores para hacer su labor académica & & & & & \\
\hline 8 & $\begin{array}{l}\text { El espacio donde trabaja es inadecuado para realizar sus las } \\
\text { labores académicas }\end{array}$ & & & & & \\
\hline 9 & $\begin{array}{l}\text { Existe hacinamiento (espacio insuficiente) en aulas o espacios } \\
\text { para realizar sus labores académicas }\end{array}$ & & & & & \\
\hline \multirow[t]{2}{*}{10} & $\begin{array}{l}\text { No cuenta con el equipo y materiales necesarios para realizar su } \\
\text { actividad académica }\end{array}$ & & & & & \\
\hline & Carga de trabajo & & & & & \\
\hline 11 & $\begin{array}{l}\text { Tiene exceso de actividades académicas o administrativas en su } \\
\text { jornada diaria de trabajo }\end{array}$ & & & & & \\
\hline 12 & $\begin{array}{l}\text { Las demandas laborales que recibe no corresponden a su nivel } \\
\text { de conocimientos, competencias y habilidades }\end{array}$ & & & & & \\
\hline 13 & Su jornada de trabajo se prolonga más de nueve horas diarias & & & & & \\
\hline
\end{tabular}




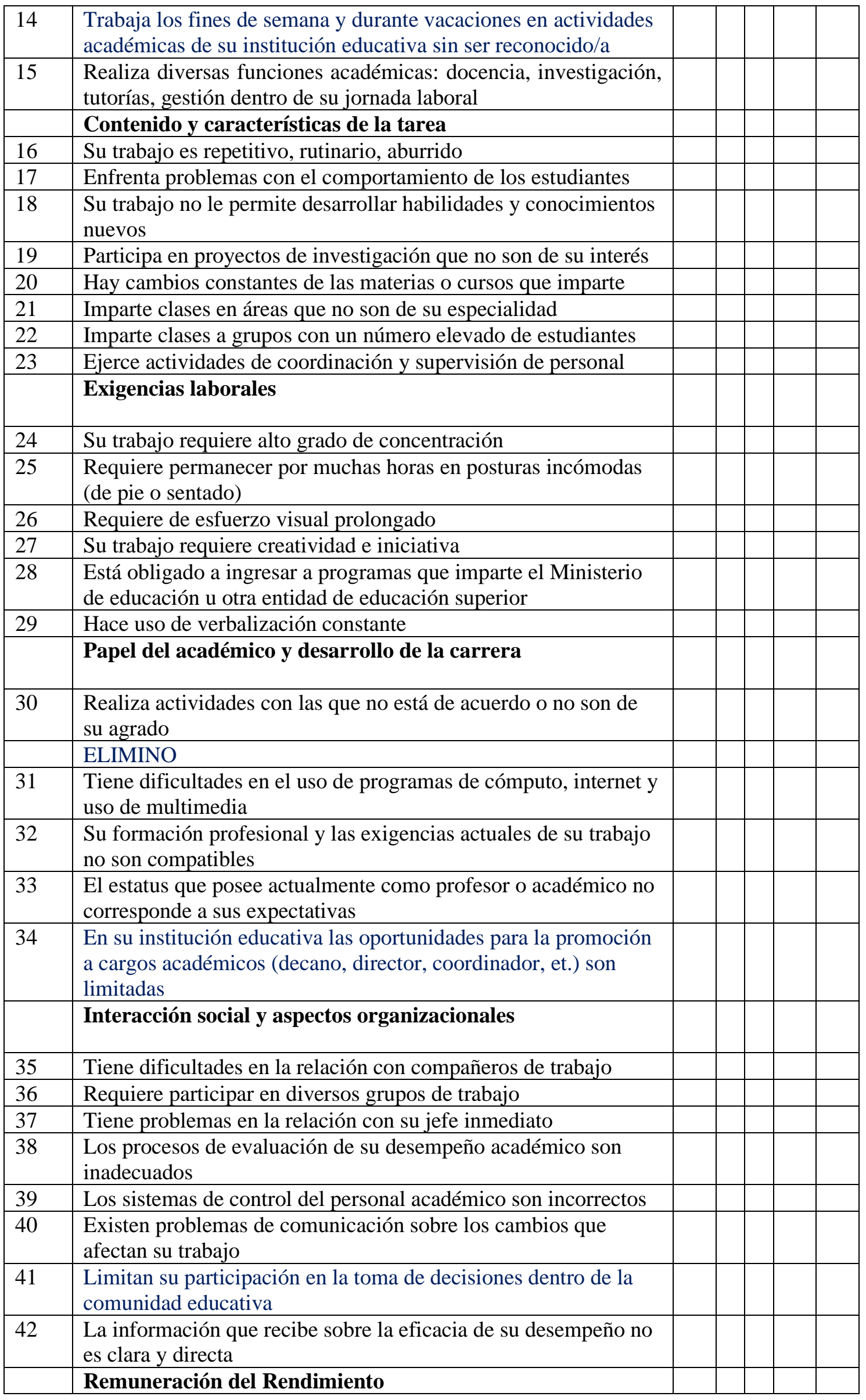




\begin{tabular}{|l|l|l|l|l|l|}
\hline 43 & $\begin{array}{l}\text { Está inconforme con el salario que recibe por el trabajo } \\
\text { académico que realiza }\end{array}$ & & & & \\
\hline & ELIMINO & & & & \\
\hline & ELIMINO & & & & \\
\hline 44 & $\begin{array}{l}\text { En su institución hay programas de estímulos económicos en } \\
\text { reconocimiento a su labor académica (por proyectos, } \\
\text { publicaciones, etc.) }\end{array}$ & & & & \\
\hline
\end{tabular}

